Proceedings of the International School and Conference on Optics and Optical Materials, ISCOM07, Belgrade, Serbia, September 3-7, 2007

\title{
Development of Low-Cost Photodynamic Therapy Device
}

\author{
N. Momchilov ${ }^{a}$, I. Bliznakova ${ }^{a, *}$, E. Borisova ${ }^{a}$, \\ P. TROYANOVA ${ }^{b}$ AND L. AVRAMOV ${ }^{a}$ \\ ${ }^{a}$ Institute of Electronics, Bulgarian Academy of Sciences \\ Sofia, Bulgaria \\ ${ }^{b}$ National Oncological Center, Sofia, Bulgaria
}

\begin{abstract}
Photodiagnosis and photodynamic therapy of non-melanoma skin cancers using delta-aminolevulinic acid/protoporphyrin IX (5-ALA/PpIX) give a combined application with broadest dissemination in the current clinical practice. The problems with using of lasers as light sources are the expenses associated with the operation of these types of installations. This is why we test the capability of cheaper sources - light-emitting diodes at $405 \mathrm{~nm}$ for fluorescence excitation of PpIX and $635 \mathrm{~nm}$ for photodynamic action initiation. A LED matrix is developed in our laboratory using two types of LEDs and a combined photodiagnosis/photodynamic theory device applicable for clinical practice is built. Geometrically matrix is formed in such way that power density at $635 \mathrm{~nm}$ is about $40 \mathrm{~mW} / \mathrm{cm}^{2}$, which allow to reach treatment doses for a 15-20 min irradiation depending of the lesion size in the focus of the system. The therapeutic mode of system developed can be used also with some other photosensitizers from the porphyrins derivatives family.
\end{abstract}

PACS numbers: 42.72.Bj, 87.64.kv, 87.19.xj

\section{Introduction}

Non-melanoma skin cancers are one of the most common malignancies worldwide. However, objective evaluation using standard techniques, such as dermatoscopy does not provide sufficient discrimination between benign and malignant forms [1]. Therefore optical techniques are applied for achievement of better diagnosis for skin neoplasia. Moreover, combinations of the therapeutic and diagnostic techniques based on the light-tissue interactions are becoming increasingly important in medicine. Photodiagnosis (PD) and photodynamic therapy (PDT) of non-melanoma skin cancers using delta-aminolevulinic acid/protoporphyrin IX

*corresponding author; e-mail: irbliznakova@abv.bg 
(5-ALA/PpIX) give a combined application with broadest dissemination in the current clinical practice $[2,3]$. For fluorescence excitation of PpIX a light source at $405 \mathrm{~nm}$ is needed, and for photodynamic action initiation illumination at $635 \mathrm{~nm}$ is required [4]. The problems with using of lasers as light sources at these two wavelengths are the expenses associated with the operation of these types of installations. Testing of the capability of cheaper sources - ultraviolet light-emitting diodes (LEDs) at $405 \mathrm{~nm}$ for fluorescence detection and light-emitting diodes with maximum of emission at $635 \mathrm{~nm}$ for photodynamic therapy of skin cancer, could give inexpensive easy-to-use systems for oncological practice. Due to the fast development of the microelectronics, powerful LEDs are currently available, which allow achievement of high power densities needed for PDT applications [5].

Similar systems for diagnostic purposes or therapeutic purposes only are developed based on filtered broad-spectra lamps $[3,6,7]$ or on diode matrices [8]. Main advantage of the system built up is the possibility for simultaneously photodetection and photodynamic treatment of the cutaneous lesion using exogenous 5-ALA/PpIX. Other advantage of the system developed is its low price, because of the LED matrix usage instead of expensive laser sources. PD/PDT device has mechanical system that allows good flexibility and possibility to reach every point of skin. This also improves the safety usage of the system in clinical practice due to non-usage of high voltage drivers. The optical characteristics of the device are suitable for PDT using aminolevulinic acid/protoporphyrin IX as a photosensitizer. The therapeutic mode can be used also with other photosensitizers from the porphyrins derivatives with similar absorption maxima around $635 \mathrm{~nm}$.

\section{Materials and methods}

New device for photodiagnosis and photodynamic therapy is built based on the light-emitting diodes used as light sources. There are two types of LEDs built in the system, these sources emit on $635 \mathrm{~nm}$ (for therapeutic applications) and on $405 \mathrm{~nm}$ (for diagnostic applications). The red emitting LEDs, applied for PDT, are described with high emitting power, which allows achieving power density up to $40 \mathrm{~mW} / \mathrm{cm}^{2}$. The system is optimized for PDT applications using aminolevulinic acid/protoporphyrin IX as photosensitizer drug.

Ninety-four LEDs type L2-0-R5TH20-1 (LedSupply Inc., USA) were chosen for building of the PDT-mode of the system developed, with total output power $1000 \mathrm{~mW}$ at $635 \mathrm{~nm}$ maximum wavelength and $\mathrm{FWHM}=15 \mathrm{~nm}$. For realization of this prototype, a universal board with parallel connected pins is used. These $5 \mathrm{~mm}$ LEDs with narrow beam divergence $\theta_{1 / 2}=20^{\circ}$ are connected to the power driver in parallel. They are built in a compact rectangular matrix $12 \times 9 \mathrm{~cm}^{2}$ with curved shape prepared in such way that the central rays of the LEDs light are crossed on $50 \mathrm{~mm}$ from the matrix surface. The geometrical positioning applied allows developing of a spot with $40 \times 50 \mathrm{~mm}$ size and power density in the spot frames is $40 \mathrm{~mW} / \mathrm{cm}^{2}$. For building of the diagnostic channel (PD-mode) 6 LEDs 
type L3-0-U5TH15-1 (LedSupply Inc., USA) were chosen. Total emitting power of the diagnostic mode is $40 \mathrm{~mW}$. The blue LEDs applied in the system have maximum of emission at $405 \mathrm{~nm}$ and FWHM $=12 \mathrm{~nm}$.

Flexible mechanical arms allow to the irradiation head of the device developed to reach easily every anatomic point of the human skin. LEDs application instead of expensive laser system improves rapidly not only the total price but the safety usage of the system developed due to non-usage of high voltage drivers. Increase in safety allows easier introducing of such developed system to clinical practice.

The system developed is introduced for initial clinical trial of the possibilities for detection and photodynamic treatment of the basal cell carcinoma lesions. Ten patients with one or more (usually two-three) lesions were treated with 5-ALA cream (ALASENSE, "Niopik" Inc., Russia) topically six hours prior PDT procedure. Before PDT treatment diagnostic channel of the system developed is used to evaluate if the ALA/PpIX is accumulated in the lesion and the long-wavelength fluorescence appeared is visualized and lesion borders are clearly evaluated by the clinician. After evaluation of the lesions therapeutic mode is switched on for 15-20 min depending from preliminary dose calculations made, related to the lesion thickness and area. In the case of incomplete removal of the neoplasia procedure could be repeated four weeks after the first treatment.

\section{Results and discussion}

\subsection{Development of the system}

A LED matrix is developed in our laboratory using two types of LEDs and a combined PD/PDT device applicable for clinical practice is built. Geometrically matrix is formed in such way that power density at $635 \mathrm{~nm}$ is about $40 \mathrm{~mW} / \mathrm{cm}^{2}$, which allows to reach treatment dose for less than $20 \mathrm{~min}$ irradiation of the lesion in the focus of the system. Doses required for PDT of basal cell carcinoma lesions are evaluated according to the lesion type and size. Usually one irradiation procedure is enough for complete treatment. Only for one of the patients treated secondary irradiation procedure was carried out due to incomplete removal of the pathology from the first PDT procedure.

Technical characteristics of the "MEDIRAY PDT" apparatus built in the frames of the research project are presented in Table. System parameters completely corresponded to the other commercialized analogues developed for the needs of photodiagnosis and photodynamic therapy in clinical practice [6-8].

For the development of user-friendly system for clinical practice to the apparatus built there were added a number of accessories, namely manual illuminator at $405 \mathrm{~nm}$, filters for light-induced fluorescence excitation and goggles for the patients and the personnel. The equipment developed was introduced for clinical trial, under guidance of Dr. Petranka Troyanova - dermatologist-oncologist in the National Oncological Center, Sofia, for diagnostics and treatment of basal cell 
TABLE

Technical characteristics of "MEDIRAY PDT" device for photodiagnosis and photodynamic therapy of skin lesions using 5-ALA/PpIX.

\begin{tabular}{l|c}
\hline \hline \multicolumn{1}{c|}{ Parameters } & Specifications \\
\hline Therapeutic field & $40 \times 50 \mathrm{~mm}$ \\
Therapeutic maximum of irradiation & $630 \mathrm{~nm}$ \\
Power density & $40 \mathrm{~mW} / \mathrm{cm}^{2}$ \\
Diagnostic maximum of irradiation & $405 \mathrm{~nm}$ \\
Object distance & $5-8 \mathrm{~cm}$ \\
Standard irradiation time & $15-20 \mathrm{~min}$ \\
Power supply & $12 \mathrm{~V}$ \\
Mass & $1 \mathrm{~kg}$ \\
Dimensions - length $\times$ width $\times$ height & \\
— whole system & $25 \times 15 \times 50 \mathrm{~cm}$ \\
— LEDs head & $20 \times 12 \times 5 \mathrm{~cm}$
\end{tabular}

carcinoma. A protocol for clinical trial was developed on the base of the literature data and existing methodological recommendations.

Typical therapeutic regime of single cutaneous malignant lesion with dimensions about $1 \mathrm{~cm}^{2}$ is as follow:

1) Ex tempora dermatologic cream is prepared using "Alasens" (Niopik Inc., Russia) using producers' prescription, which is bring on the pathological zone and on 5-6 $\mathrm{mm}$ around on the healthy skin surface. The all zone of interest is covered by non-transparent bandage;

2) After six hours a diagnostic analysis is carried out using blue light irradiation of the skin. Fluorescent signal is detected through high-pass filter and allows visualization of lesions and clarification of their borders, with sensitivity better that with dermatoscopic evaluation of the skin;

3) Tumor site is irradiated with a single procedure for about $15 \mathrm{~min}$ in the frames of working field of the apparatus (on $5 \mathrm{~cm}$ distance from the irradiation plane).

During the therapeutic procedure patient has some sensations, which vary from slight warming to weak pain. Lesion become moist and zone around is turned red. There are not observed other undesirable side effects. After one week a cutaneous lesion recovery is observed, and a full healing of the skin is observed after about a month without scars or cicatrices. This treatment could be repeated if needed without any complications for the patient if partial healing is achieved in the first therapeutic procedure. 


\subsection{Clinical cases}

Nevertheless that according to existing literature 5-ALA-assisted photodynamic therapy is applied for broad range of benign and malignant dermatological lesions [2-7], the main application in the clinical practice is related to the basal cell carcinoma therapy. This skin lesion is the broadest progress of this kind of cutaneous tumor, as well as high-rate of recurrences using standard therapeutic methods.

In Fig. 1 there are presented possibilities of the photodynamic therapy after single treatment procedure using apparatus developed in the frames of our work. One of the basal cell lesions of a patient with a multiple carcinoma (Fig. 1a) is treated using 5-ALA. Control clinical examination three months after the PDT procedure reveal healthy skin with slight cosmetic defect (Fig. 1b).
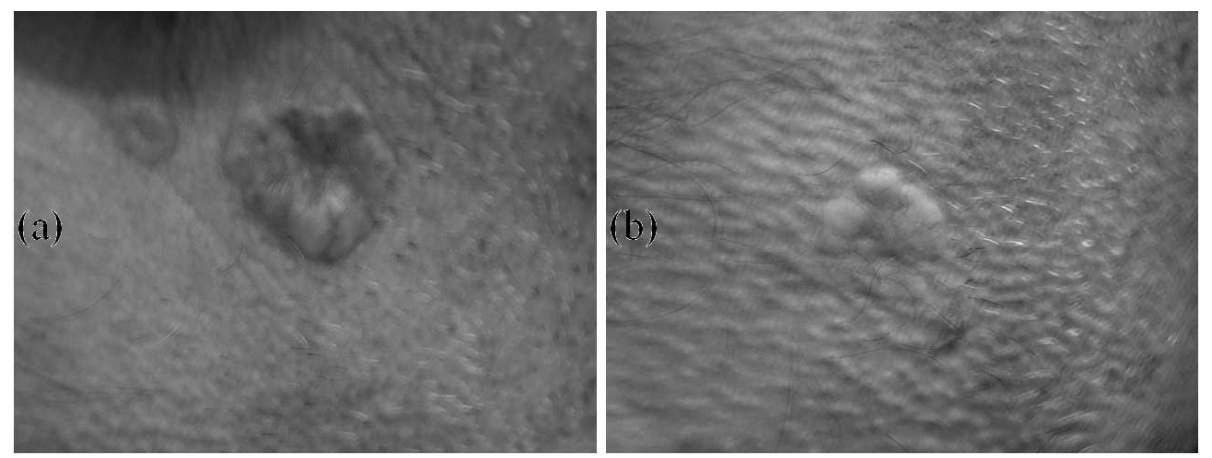

Fig. 1. One lesion from the patient with a multiple basal cell carcinoma before PDT (a) and three months after PDT (b).

Except good therapeutic results received during clinical trial the role of fluorescent diagnosis was highly appreciated by the clinicians. Fluorescence imaging has been shown to be a potential complement to visual inspection for demarcation of basal cell carcinoma lesions [7]. Promising results are obtained for the cases of addition of exogenous fluorescent signal to the autofluorescence from the lesion. Adding of aminolevulinic acid allows increasing rapidly the contrast and rising of the possibilities for precise detection of all lesions in the skin of the patient. In two of the cases investigated only one lesion was diagnosed clinically, but application of 5-ALA reveal additional small lesions near to the observable one.

\section{Conclusions}

In conclusion, we have investigated the possibility to discriminate tumor areas from normal skin using fluorescence of 5-ALA/PpIX for basal cell carcinoma lesions using excitation at $405 \mathrm{~nm}$. The fluorescence detection is combined with therapeutic mode - using PDT effect induced during irradiation of protoporphyrin IX in the red spectral region around $630 \mathrm{~nm}$. For the needs of clinical 
practice we constructed appropriate LED-based device with both diagnostic and therapeutic modes. This inexpensive prototype is described using a dual wavelength concept for either fluorescence excitation or photodynamic treatment to allow detection and treatment of basal cell carcinoma lesions using 5-ALA/PpIX as a fluorescent marker as well as photosensitizing compound. The low price, portability, good flexibility and improved safety of the device developed make it affordable not only for big hospitals, but for small clinics and doctor's offices.

\section{Acknowledgments}

This work was supported by the Bulgarian Ministry of Education and Science under grant MUF-03/05 "Development of apparatus and methods for optical biopsy of human skin".

\section{References}

[1] U. Wollina, C. Nelskamp, A. Scheibe, D. Fassler, W. Schmidt, Skin Res. Tech. 13, doi: 10.1111/j.1600-0846.2007.00252.x (2007).

[2] M. Pierre, E. Ricci, A. Tedesco, M. Bentley, Pharmaceut. Res. 23, 360 (2006).

[3] F. Fischer, E. Dickson, R. Pottier, H. Wieland, Lasers Med. Sci. 16, 199 (2001).

[4] A. Katzir, Lasers and Optical Fibers in Medicine, Academic Press, San Diego (CA) 1993.

[5] E. Borisova, B. Vladimirov, I. Angelov, L. Avramov, Proc. SPIE 6604, 6604-2H (2007).

[6] F. Fischer, E. Dickson, J. Kennedy, R. Pottier, Lasers Med. Sci. 16, 207 (2001).

[7] M. Ericson, J. Uhre, C. Strandeberg, B. Stenquist, O. Larko, A. Wennberg, A. Rosen, J. Biomed. Opt. 10, 034009 (2005).

[8] http://www.biospec.ru/. 